\title{
Silicato Tricálcico en Endodoncia Compleja
}

\author{
Tricalcium Silicate in Complex Endodontic
}

Maximiliano Casa Herzmann ${ }^{1}$ \& Julieta Anllo²

\begin{abstract}
CASA, H. M. \& ANLLO, J. Silicato tricálcico en endodoncia compleja. Int. J. Odontostomat., 15(4):948-952, 2021.
RESUMEN: En casos complejos como el que se presenta, es necesario detallar cada una de las dificultades que se debe sortear, para brindar al diente la posibilidad de seguir formando parte del sistema estomatognático. Para planificar un tratamiento endodóntico integral es necesario evidenciar la complejidad de los diferentes ítems: desde el retiro del instrumento separado en el conducto distal, la localización del conducto mesiolingual parcialmente calcificado, la desobturación del conducto mesiobucal, el sellado de la perforación en la zona de la furcación y la limpieza exhaustiva del sistema de conductos radiculares, así, se le entrega a la biología, el tiempo necesario para reaccionar y reparar las lesiones osteolíticas presentes tanto a nivel de la furcación como en apical en ambas raíces; para, por último, rehabilitar el diente devolviéndole su funcionalidad y estética. En este caso clínico, se aprecia la perforación en la zona de la furcación, a nivel de la cresta ósea en el tercio corono radicular. Cuanto mayor es el tamaño de la perforación menor posibilidad de éxito. En la actualidad, con el empleo delos nuevos biomateriales de obturación y sellado endodóntico, como los cementos biocerámicos de última generación, se logra mejorar los aspectos clínicos, mecánicos y biológicos, con la posibilidad de realizar procedimientos que antes eran impensados, mejorando así su pronóstico.
\end{abstract}

PALABRAS CLAVE: endodoncia compleja, sellado de perforación, biocerámico, microscopia, biocerámico, biodentine, bioroot.

\section{INTRODUCCIÓN}

El éxito en tratamientos complejos radica en una planificación multidisciplinaria, donde se debe exponer cada reto a sortear y evaluar científicamente la probabilidad de sobrevida. La habilidad del operador no se debe tomar a la ligera, ya que múltiples estudios sugieren que con un tratamiento en manos de un especialista se obtiene mejores posibilidades que en manos inexpertas.

El $47 \%$ de las perforaciones iatrogénicas se crean durante el tratamiento endodóntico y el $53 \%$ se realizan en el tratamiento prostodóntico siendo los dientes maxilares $(74,5 \%)$ frecuentemente más afectados que los dientes mandibulares (25,5\%) (Jitaru·et al., 2016).

De acuerdo a la localización, tiempo transcurrido, presencia o ausencia de infección y tamaño de la perforación, Fuss \& Trope (1996) aconsejan al operador en lo que respecta al pronóstico inmediato y mediato. Se puede orientar al profesional acerca del pro- nóstico de dicho tratamiento. Las perforaciones mayores de $3 \mathrm{~mm}$ tienen un peor pronóstico (Parirokh et al., 2017), al igual que las que se tratan de forma tardía.

En la década de los 90, Lee y Torabinejad et al., desarrollaron un cemento conocido con el nombre de agregado de trióxido mineral (MTA). No todos los materiales que se emplean en el tratamiento de las perforaciones radiculares tienen el mismo mecanismo de fraguado, pues mientras unos tienen sólo una actividad selladora, otros son bioactivos y se caracterizan por ser capaces de inducir mineralización en el tejido óseo y la formación de tejidos duros en el diente (Gandolfi et al., 2011).

La disociación de los iones de calcio e hidroxilo al momento del fraguado del biocerámico aumenta el $\mathrm{pH}$ de la solución lo que promueve un ambiente desfavorable para el crecimiento bacteriano (Han \& Okiji, 2013), con menor tiempo de fraguado en compara-

\footnotetext{
${ }^{1}$ Programa de Especialización en Endodoncia, Universidad Autónoma de Chile, Chile.

${ }^{2}$ Carrera de Odontología, Cátedra Integral del Niño, Universidad Autónoma de Chile, Santiago, Chile.
} 
ción con el resto de los materiales analizados (Parirokh et al.) y controla la pureza del silicato de calcio del Biodentine, con lo cual se obtiene como resultado un menor nivel de porosidad y una mayor resistencia mecánica; agregando al líquido una sustancia encargada de reducir el agua (polímero hidrosoluble), con el fin que permanezca el equilibrio entre la mezcla y la consistencia de agua, presentando de esta manera una mayor resistencia a la compresión y flexión que el MTA, llegando a ser similar a la dentina (Cedrés et al., 2014).

El menor tiempo de fraguado se traduce en una menor posibilidad de contaminación bacteriana (Baroudi \& Samir, 2016). Biodentine es capaz de inducir la diferenciación de las células madre mesenquimales y de la pulpa dental en células osteoblásticas y odontoblastos responsables de la dentinogénesis reparativa (Rajasekharan et al., 2014).

\section{CASO CLÍNICO}

Se presentó a la consulta una paciente sexo femenino, 33 años, ASA I, con dolor leve en un diente 3.6, tratado endodónticamente hace 3 años, cuyo diagnóstico fue de periodontitis apical asintomática. Los test de sensibilidad resultaron negativos para las pruebas térmicas, y positivos a las de percusión. En la clínica se observó una gran destrucción coronaria, apreciando una comunicación mediana desde el piso de la cámara pulpar hacia el ligamento periodontal a nivel da la furcación. Dicho diente se localizaba con una ligera giroversión. Radiográficamente se observó una lesión radiolúcida apical en ambas raíces y en la zona de la furcación. Una obturación incompleta en el conducto mesiobucal, el conducto mesiolingual parcialmente calcificado y una lima separada en el conducto distal.

Como en todo tratamiento, el análisis clínico e imagenológico (Figs. 1 y 2) es fundamental no solo para empezar a evidenciar las posibles complicaciones técnicas y biológicas al momento de afrontar dicho tratamiento, sino para pronunciarse desde el punto de vista del pronóstico, como lo recomiendan las directrices de la valoración de la dificultad y riesgo endodóntico de la Asociación Americana de Endodoncia.

Con el empleo del microscopio clínico y del ultrasonido, se localizaron ambos conductos mesiales.

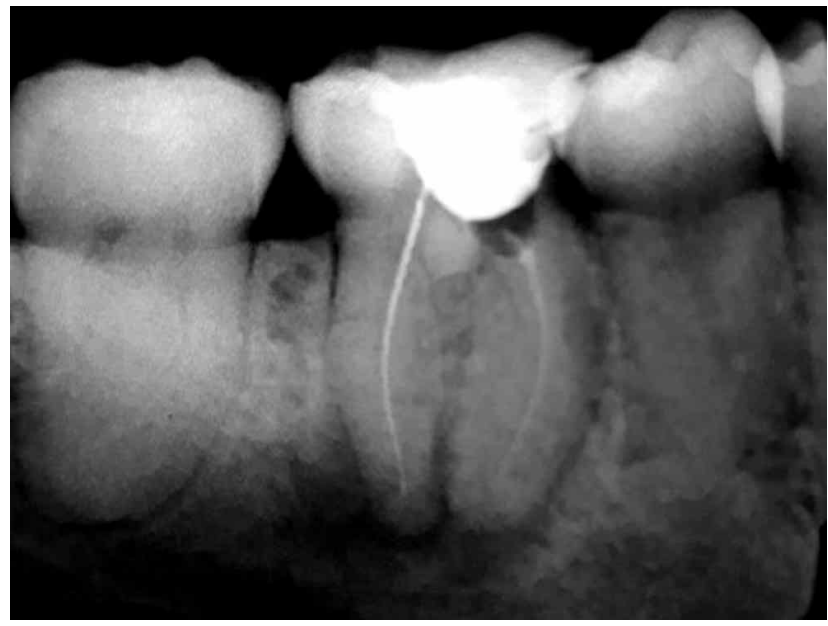

Fig. 1. Análisis radiográfico preoperatorio. Se observa una lesión radiolúcida apical en ambas raíces y en la zona de la furcación. Una obturación incompleta en el canal mesiobucal, el canal mesiolingual parcialmente calcificado y una lima separada en la raíz distal.

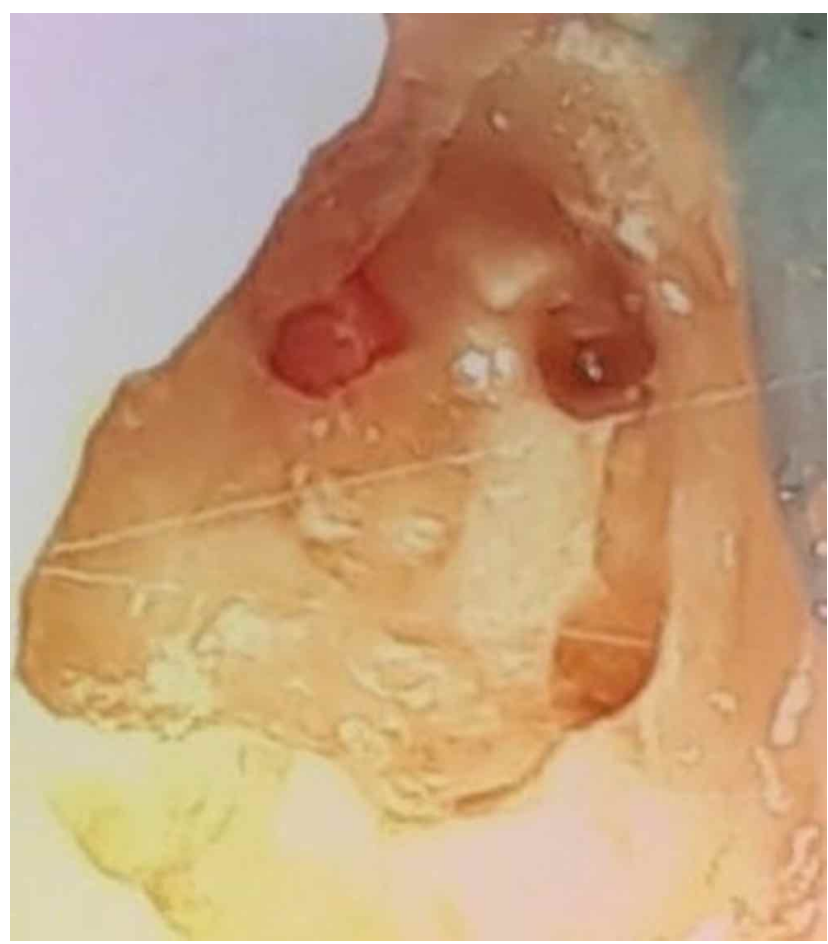

Fig. 2. Análisis clínico, evidenciando la perforación a nivel del piso de la cámara pulpar y la falta de localización del canal mesiolingual.

Una vez localizados, permeabilizados y preparados química y mecánicamente con el empleo de hipoclorito de sodio al 5,25\% e instrumental mecanizado; se procedió a la obturación de forma definitiva con cemento a base de biocerámicos (BioRoot, Septodont) y conos de gutapercha, empleando la téc- 
nica de condensación lateral a conos múltiples. Conjuntamente se comenzó el proceso de remoción del instrumento separado en el conducto distal (Figs. 3 y 4).

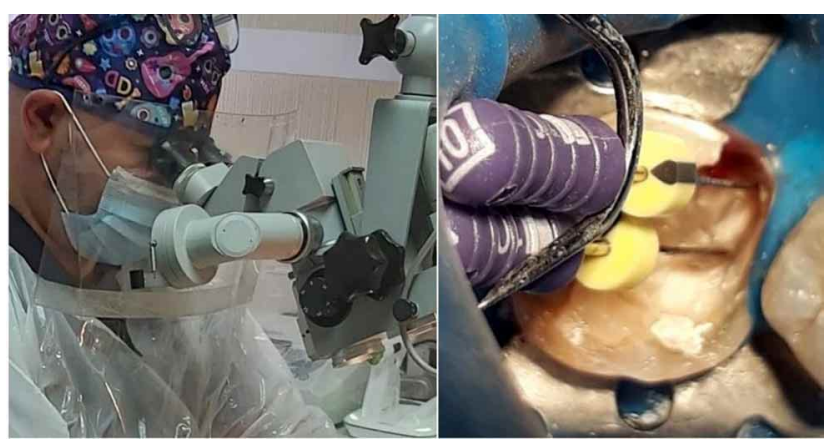

Fig. 3. Localización del canal mesiolingual con magnificación y ultrasonido.

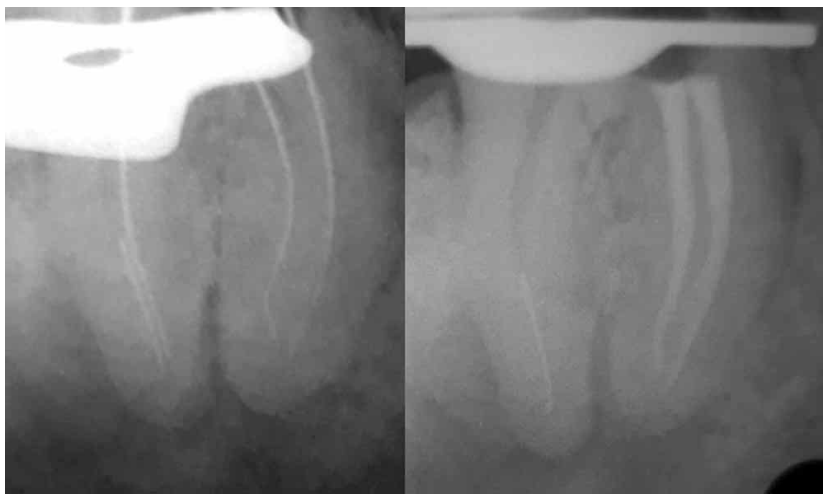

Fig. 4. Odontometría y obturación de las raíces mesiales con BioRoot, (Septodont, Saint-Maur-des-Fossés, France).

Durante el proceso de remoción del instrumento separado, el mismo se volvió a separar por debajo del tercio medio. Nuevamente la tecnología resultó indispensable en estos procedimientos, logrando identificar la lima residual y posteriormente facilitando su remoción (Fig. 5), registrando radiográficamente dicha maniobra (Fig. 6).

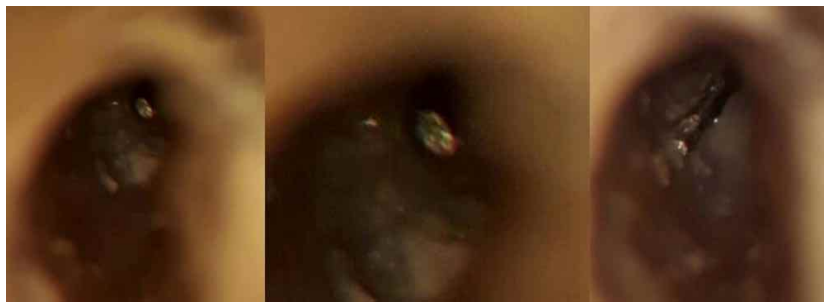

Fig. 5. Se visualiza el extremo coronal de la lima con la ayuda de la magnificación.

Luego de irrigar profusamente con hipoclorito al 5,25\% el conducto distal se decidió obturar el mismo empleando la técnica hidráulica con cemento a

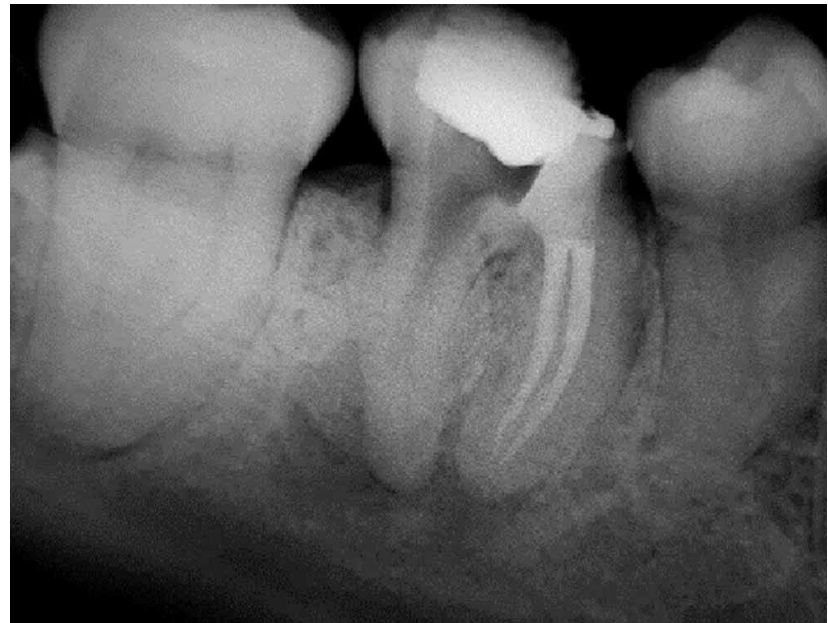

Fig. 6. Control radiográfico de la lima removida.

base de biocerámico (BioRoot, Septodont, Saint-Maurdes-Fossés, France) y conos de gutapercha utilizando la técnica de condensación lateral a conos múltiples (Fig. 7).

Planificando con antelación la rehabilitación final, y previsto el debilitamiento que ocasionaría el empleo del ultrasonido, se procedió a cementar un poste de fibra en el conducto distal para colaborar así con la confección del muñón protésico. Posteriormente, se selló la perforación con un biocerámico (Biodentine, Septodont, Saint-Maur-des-Fossés, France) aplicando las mejores características biológicas y mecánicas buscadas;no solo bioactividad, biocompatibilidad, e inductor del tejido mineralizado sino una gran resistencia mecánica compresiva y torsional, semejante a la dentina. Todos los pasos clí-

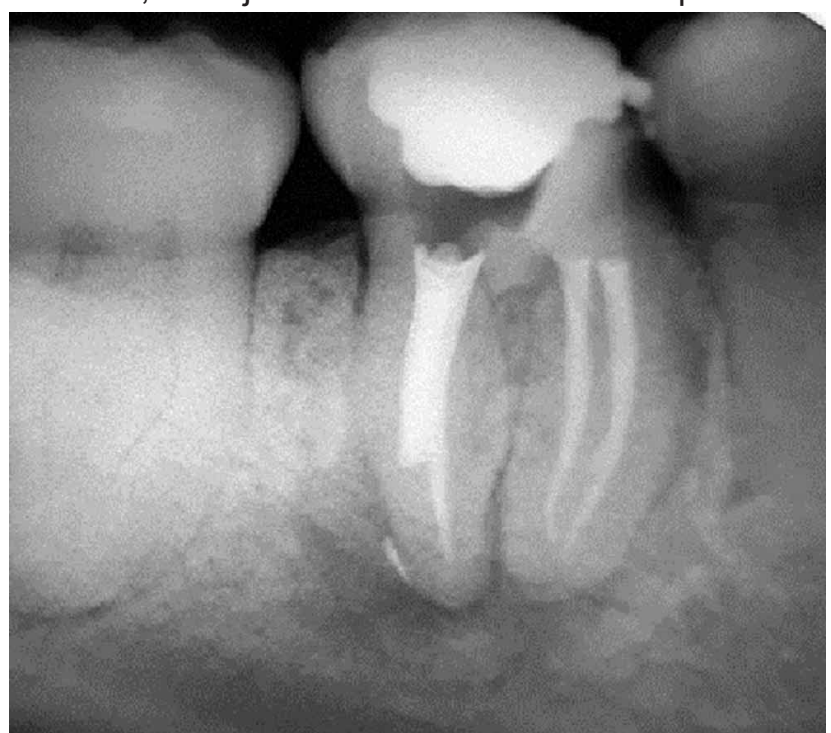

Fig. 7. Control radiográfico de la obturación. 
nicos se verificaron radiográficamente. Se talló un muñón mixto entre resina, poste de fibra, dentina remanente y Biodentine (Fig. 8)

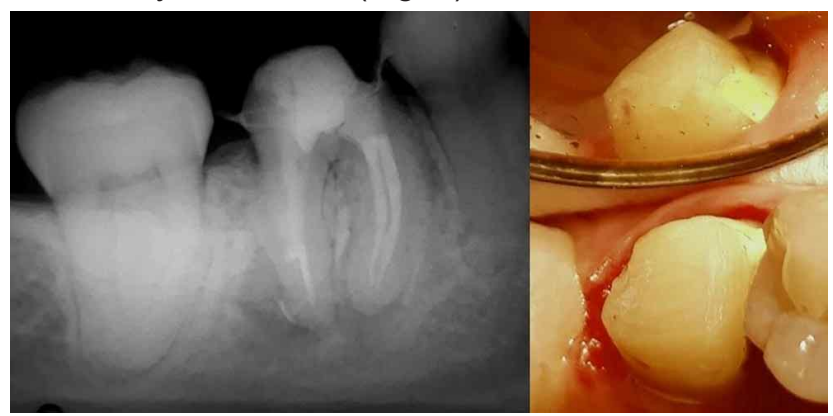

Fig. 8. Control radiográfico inmediato a la obturación, y clínico a la preparación para alojar su PFU.

Posteriormente se cementó la prótesis fija unitaria (PFU) evaluando la oclusión y salud gingival. Como bien decía el Prof Dr. Maisto, en su calidad de experto en la materia "Podremos hablar de éxito en un tratamiento endodóntico, solo cuando en los controles a distancia, clínica y radiográficamente así se vea," (Maisto, 1982) (Fig. 9).

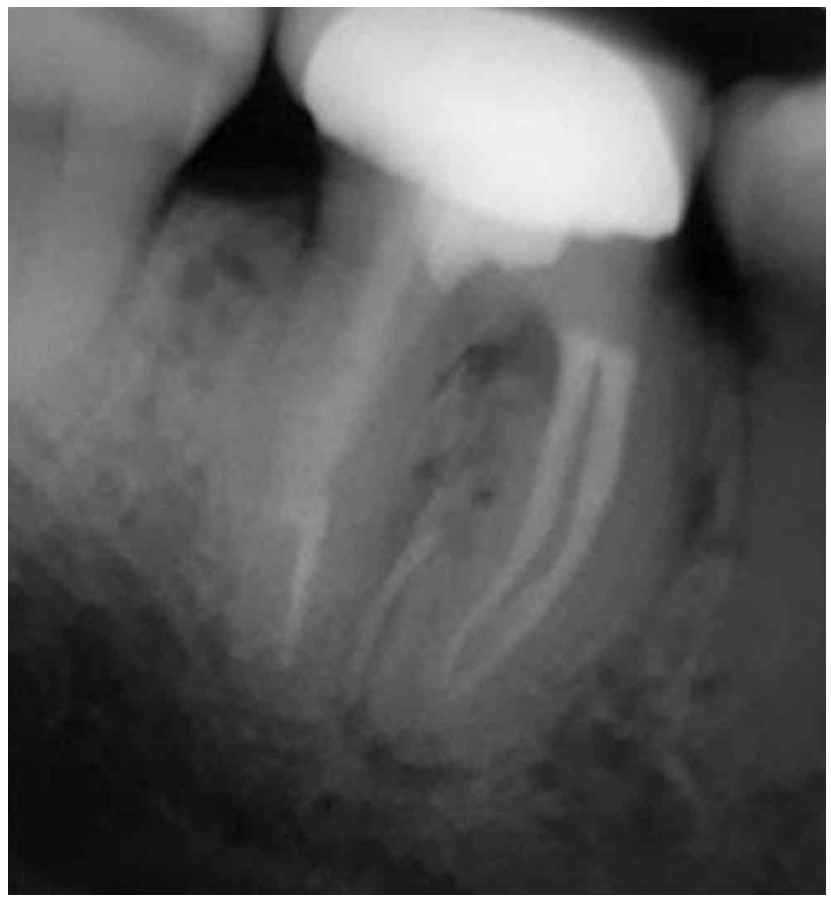

Fig. 9. Control a los 4 años.

\section{DISCUSIÓN}

Siendo un caso excepcionalmente complejo desde el punto de vista endodóntico, biológico y rehabilitador;el éxito de éste radica en realizar una correcta valoración de riesgo basado en un exhaustivo estudio clínico y radiográfico previo, desde el punto de vista biológico y tecnológico, pasando por las habilidades requeridas para afrontar el mismo.

La elección del enfoque para la terapia depende de la evaluación de los factores relacionados con la perforación tales como la ubicación, acceso, región de visualización (caras libres o proximales), el tamaño y la presencia o ausencia de pérdida ósea en la zona adyacente a la perforación, el compromiso estético y periodontal, biotipo gingival, el costo/ beneficio/riesgo y las expectativas del paciente (Pereira, 2008)

El tamaño de la perforación es muy importante para el pronóstico. Cuanto mayor sea la perforación menos éxito se espera del tratamiento (Fuss \& Trope; Tsesis \& Fuss, 2006). Mantener en cada etapa el control bacteriano es todo un reto, ya que en ocasiones se deben realizar maniobras tempranas para lograr una aislación absoluta correcta y por demás obligatoria.El uso del dique de goma es de especial importancia para el tratamiento expuesto, puesto que garantiza un medio libre de humedad que evite la contaminación bacteriana proveniente de la saliva, debido a que esta puede invadir el complejo dentinopulpar y conllevar a la infección (Accorinte et al., 2006; Marques et al., 2015).

El microscopio operatorio ha demostrado, comportarse como un perfecto aliado del dentista con práctica de endodoncia, pasando de ser un instrumento complementario o auxiliar a convertirse en el coprotagonista del éxito de un tratamiento de conductos (Moradas Estrada, 2017).

El conocimiento de las características propias de los nuevos biomateriales como el Biodentine (Cedrés et al.) hace posible no solo sellar la perforación existente (Gandolfi et al.), sino reforzar las estructuras radiculares y coronales debilitadas, siendo la base para una futura rehabilitación, respetando todo aquel principio de espacio y convivencia entre la (PFU) prótesis fija unitaria, la encía, hueso y espacios libres.

El compromiso de dichas acciones radica en brindarle al cuerpo el medio adecuado y el tiempo necesario para responder y reparar, las lesiones existentes no solo en la zona de la perforación, sino en apical de ambas raíces (Fuss \& Trope). 
La curva de aprendizaje necesaria para emplear magnificación y el ultrasonido debe ser excluyente. No es aconsejable simplemente hacerse de un medio de magnificación y de un momento a otro, comenzar a emplearlo sobre un paciente sin laformación apropiada. Los movimientos y las diferentes técnicas para hacer de la magnificación y el ultrasonido, implementos necesarios e indispensables, nos obliga a prepararnos con antelación (Moradas Estrada).

CASA, H. M. \& ANLLO, J. Tricalcium silicate in complex endodontics. Int. J. Odontostomat., 15(4):948-952, 2021.

ABSTRACT: In cases as complex as the one that is presented, it is necessary to detail each of the difficulties that must be overcome, to give the tooth the possibility of continuing to form part of the stomatognathic system. To plan a comprehensive endodontic treatment, it will be necessary to demonstrate the complexity of the different items present: from the removal of the file separated from the distal canal, the location of the calcified mesiolingual canal, the deobturation of the mesiobuccal canal, the sealing of the perforation in the area of the furcation, up to the exhaustive cleaning of the root canal system, are important to give biology the time necessary to repair the osteolytic lesions present both at the level of the furcation and apically in both roots; to finally rehabilitate the tooth, restoring its functionality and aesthetics.In this particular case, the perforation can be seen in the furcation area, at the level of the bone crest in the Root crown third. The larger the piercing, the less success is expected of the treatment. Thanks to the use of technologies applied to obtain new endodontic filling and sealing materials such as the latest generation of bioceramic cements, clinical, mechanical and biological aspects are improved, providing the possibility of performing procedures that were previously unthinkable, thus improving prognosis.

KEY WORDS: complex endodontics, perforation sealing, bioceramic, microscopy, bioceramic, biodentine, bioroot.

\section{REFERENCIAS BIBLIOGRAFICAS}

Accorinte, M.; Reis, A.; Loguercio, A.; De Araüjo, V. \& Muench, A. Influence of rubber dam isolation on human pulp responses after capping with calcium hydroxide and an adhesive system. Quintessence Int., 37(3):20512, 2006.

Baroudi, K. \& Samir, S. Sealing ability of MTA used in perforation repair of permanent teeth; literature review. Open Dent J., 10(1):278-86, 2016.

Cedrés, C.; Giani, A. \& Laborde, J. C. Una nueva alternativa biocompatible: BIODENTINE. Actas Odontol., 11(1):11-6, 2014.

Fuss, Z. \& Trope, M. Root perforations: classification and treatment choices based on prognostic factors. Endod. Dent. Traumatol., 12(6):255-64, 1996.
Gandolfi, M. G.; Taddei, P.; Siboni, F.; Modena, E.; De Stefano, E. D. \& Prati, C. Biomimetic remineralization of human dentin using promising innovative calcium-silicate hybrid "smart" materials. Dent. Mater., 27(11):1055-69, 2011.

Han, L. \& Okiji, T. Bioactivity evaluation of three calcium silicatebased endodontic materials. Int. Endod. J., 46(9):808-14, 2013.

Jitaru, S.; Hodisan, I.; Timis, L.; Lucian, A. \& Bud, M. The use of bioceramics in endodontics - Literature review. Clujul Med., 89(4):470, 2016.

Maisto, O. A. Endodoncia. $4^{\mathrm{a}}$ ed. Ciudad de Buenos Aires, Mundi, 1982.

Marques, M. S.; Wesselink, P. R. \& Shemesh, H. Outcome of direct pulp capping with mineral trioxide aggregate: a prospective study. J. Endod., 41(7):1026-31, 2015.

Moradas Estrada, M. Importance of the magnificent in conservative dentistry: bibliographic review. Av. Odontoestomatol., 33(6):28393, 2017.

Parirokh, M.; Torabinejad, M. \& Dummer, P. M. H. Mineral trioxide aggregate and other bioactive endodontic cements: an updated overview - part I: vital pulp therapy. Int. Endod. J., 51(2):177205, 2017.

Pereira, L. A. P.; Cardoso, R. J. A. \& Motta, R. H. O tratamento das perfurações radiculares. Atualização clínica em Odontología. Antonio Carlos Bombana. São Paulo, Artes Médicas, 2008. pp.104-15.

Rajasekharan, S.; Martens, L. C.; Cauwels, R. G. E. C. \& Verbeeck, R. M. H. BiodentineTM material characteristics and clinical applications: a review of the literature. Eur. Arch. Paediatr. Dent., 15(3):147-58, 2014

Tsesis, I. \& Fuss, Z. Diagnosis and treatment of accidental root perforations. Endod. Top., 13(1):95-107, 2006.

Direccion para correspondencia:

Prof. Maximiliano Casa, Herzmann, CD, MSc

Juan Egaña 1873

Especialidad en Endodoncia

Universidad Autónoma de Chile

Temuco

CHILE

E-mail: Maximiliano.casa@uautonoma.cl

\section{2}

